\title{
Quantitative Electron-probe Microanalysis and WDS Background Measurement
}

\author{
P. K. Carpenter ${ }^{1}$ and J. J. Donovan ${ }^{2}$ \\ ${ }^{1}$ Dept. of Earth and Planetary Sciences, Washington University in St. Louis, Campus Box 1169, Saint \\ Louis, MO, 63130, USA \\ ${ }^{2}$ CAMCOR, University of Oregon, Eugene, OR 97403, USA
}

The accuracy of electron-probe microanalysis (EPMA) by wavelength-dispersive spectrometry (WDS) depends critically on spectrometer alignment, specimen positioning, pulse-height (PHA) discrimination, and deadtime correction, but also on the accurate measurement of characteristic x-ray intensities (i.e., "peak") and continuum (i.e., "background") which form the k-ratio that is converted by correction algorithms to yield concentration. Using pure elements, oxides, and end-member silicate minerals as primary standards minimizes errors in the selection of WDS background offsets for calibration, whereas the diverse element inventory of samples presents possible interferences for either peak or background due to $\mathrm{n}^{\text {th }}$-order diffracted lines from these elements. The use of WDS wavelength scans acquired (using elevated probe current and long count times) on pure elements, primary and secondary standards, and selected samples is valuable in identifying background offsets and assessing the analytical setup. Analysis of secondary standards helps identify systematic errors and is mandatory for quality control.

WDS backgrounds must be chosen to avoid first-order interferences such as the L-lines of Cd, Sb, and Te shown in Figure 1. If $\mathrm{Cd}$ is measured using an Ar P-10 detector, visual inspection reveals that the Ar $\mathrm{K}$-edge is located between $\mathrm{Cd} \mathrm{L} \alpha_{1}$ and the low-side background position and effectively negates use of that background. Indeed, differential absorption by Ar results in the $\mathrm{Cd} L \beta_{1}$ peak having higher intensity than $\mathrm{Cd} \mathrm{L} \alpha_{1}$ Graphical scan data represent the peak width and relative intensity better than tabulated peak positions and especially illustrates the magnitude of absorption edge effects. On-peak interferences (e.g., Te $\mathrm{L \eta}$ on $\mathrm{Sb} \mathrm{L} \alpha_{1}$ ) require an interference correction as part of the ZAF iteration loop. A WDS scan on $\mathrm{Sb}_{2} \mathrm{Te}_{3}$ would not reveal this built-in interference, thus underscoring the importance of pure element scan data. The use of $\mathrm{Sb}_{2} \mathrm{Te}_{3}$ as a standard to analyze Te-free samples would result in underestimation of $\mathrm{Sb}$ without the interference correction that would itself require an $\mathrm{Sb}$-free $\mathrm{Te}$ standard. This set of elements thus illustrates the central role of WDS scan data in understanding the details of x-ray measurement.

For a given analytical line, how does the background intensity vary as a function of composition, how does one determine the "true" background, detect low levels of that element in materials with confidence, and also detect the many high order background interferences in a systematic way? These questions are not easily answered with individual WDS scans. The mean atomic number (MAN) background method utilizes measurement at the peak position on a suite of standards known or presumed to be free of that element; a fit of average $Z$ vs. absorption-corrected intensity provides a powerful tool for analysis [1]. In Figure $2 \mathrm{~A}$ and B, MAN plots for Manganese $\mathrm{K} \alpha$ and Phosphorus $\mathrm{K} \alpha$ measured on a suite of silicate standards reveals the presence of $\mathrm{Mn}$ in a secondary wollastonite standard, and undocumented low level $\mathrm{P}$ in Kakanui hornblende. The standards free of these elements establish the "true" background, and it is possible to detect $\mathrm{Mn}$ and $\mathrm{P}$ at trace levels with improved confidence as the points plot above the background fit. In Figure $2 \mathrm{C}$, the MAN data for Fluorine K $\alpha$ reveals the detection of high-order peaks (or their tails) that have intensity at the fluorine peak position. It would otherwise require inspection of numerous WDS scans to reveal these interferences. The MAN plots therefore provide important information regarding the concentration of an element in an internally consistent manner by comparison to all measured standards as well as peak interferences from other elements. 
MAN data that plot below the fit line may indicate a systematic error in the absorption correction of intensity data. Figure $2 \mathrm{D}$ illustrates the range of calculated intensity data for Sulfur $\mathrm{K} \alpha$ in $\mathrm{Zr}$ and $\mathrm{Cd}$ matrices. Sulfur Ka is very close to the Zr L3 absorption edge and there is poor agreement between the mass absorption coefficients (MAC) for S Ka by $\mathrm{Zr}$ using different MAC data sets. The MAC for S K $\alpha$ by $\mathrm{Cd}$ does not show such disagreement but suggests that problems may exist in the measurement of Cd-bearing sulfides, for example. Note that these measurements are made at the $\mathrm{S} K \alpha$ peak position on pure elements so that actual alloy compositions are not required. It is therefore possible to identify analytical problems in compositional systems using pure elements. We continue to evaluate these methods and their role in quantitative EPMA.
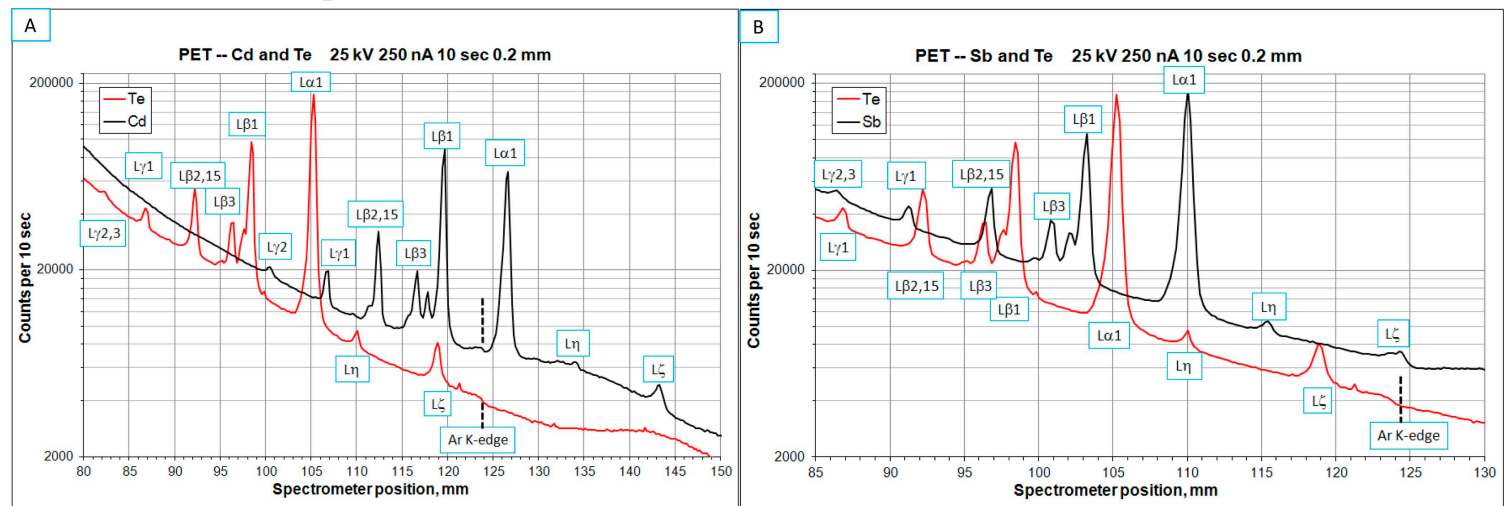

Fig. 1 Wavelength scans on pure elements for background selection, obtained using PET crystal with P-10 flow detector. A: Dramatic effect of Ar K-edge differential absorption on intensity of Cd L $\beta$ family relative to $\mathrm{Cd} L \alpha_{1}$ and problem of low-side background selection for $\mathrm{Cd} L \alpha_{1}$, also partial overlap of $\mathrm{Cd} L \gamma_{1}$ on $\mathrm{Te} \operatorname{L} \alpha_{1}$. B: Pathological interferences for Te and $\mathrm{Sb}$ with mutual background interferences and on-peak Te L $\eta$ interference on $\mathrm{Sb} \mathrm{L} \alpha_{1}$. Visual inspection of wavescan provides clues to proper setup.

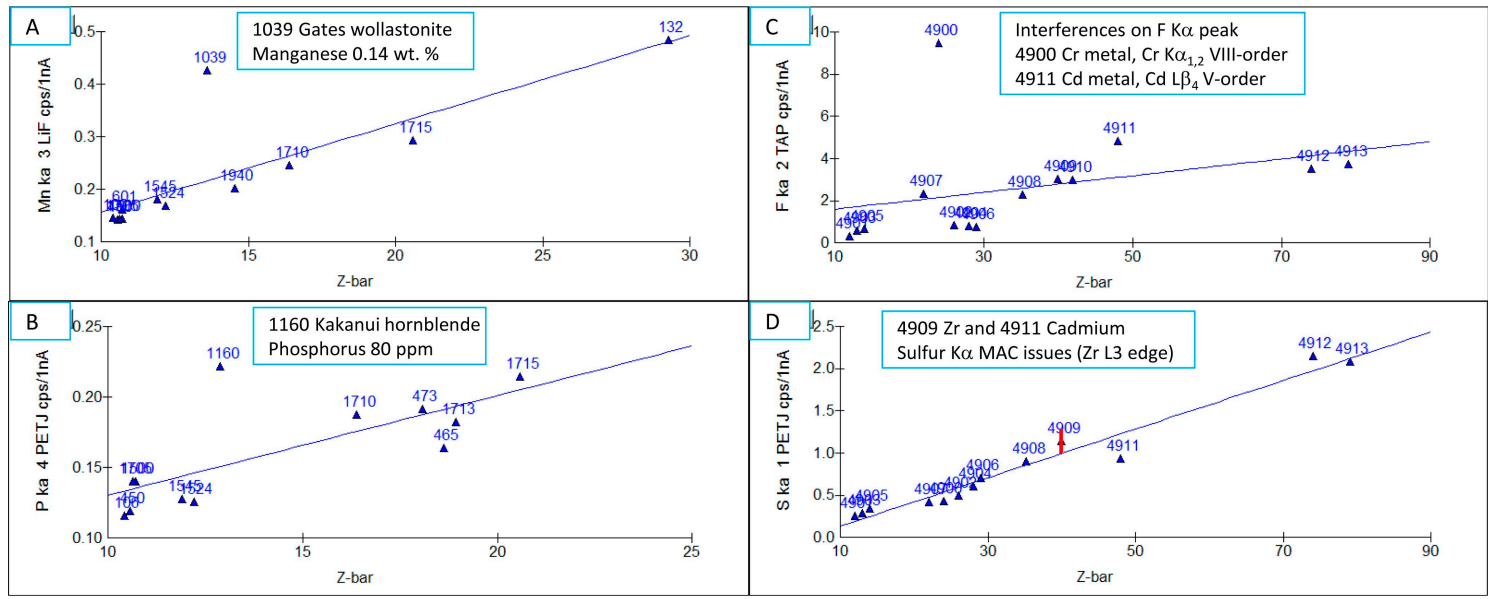

Fig. 2 MAN plots. A and B: MAN plots for Mn $\mathrm{K} \alpha$ and $\mathrm{P} \mathrm{K} \alpha$ on Gates wollastonite and Kakanui hornblende indicating the presence of undeclared concentrations for these elements and confirming the internal consistency of the suite of silicate and oxide standards used for calibration. C: Peak interferences on F K $\alpha$ using TAP crystal indicating effect of high-order reflections from $\mathrm{Cr}\left(\mathrm{Cr} \mathrm{K} \alpha_{1,2} \mathrm{VIII}^{\text {th }}\right.$ order $)$ and $\mathrm{Cd}\left(\mathrm{Cd} \mathrm{L} \beta_{3,4} \mathrm{~V}^{\text {th }}\right.$ order$)$. D: MAN data for $\mathrm{S} \mathrm{K} \alpha$ background determined on suite of pure metals. Uncertainties in mass absorption coefficient for $\mathrm{S}$ $\mathrm{K} \alpha$ by $\mathrm{Zr}$ (red line shows range of intensities resulting from use of available MACs for $\mathrm{S}$ K $\alpha$ which is very close to $\mathrm{Zr} \mathrm{L} 3$ absorption edge).

\section{References}

[1] Donovan, J. J. and Tingle, T. N. JMSA, 2, No.1, 1996. 